DOI: 10.12731/2227-930X-2016-2-79-87

\title{
ПРИНЯТИЕ РЕШЕНИЯ О ФОРМЕ ДИФРАКЦИОННЫХ СТРУКТУР В ЗАДАЧЕ РАССЕЯНИЯ РАДИОВОЛН
}

\author{
Преображенский А.П., Чопоров О.Н.
}

В работе рассматривается задача рассеяния электромагнитных волн на различных дифракиионных структурах. Решение задачи рассеяния осуществляется на основе метода интегральных уравнений. По диаграммам обратного рассеяния при разных частотах падающей волны принимается решение о форме исследуемого объекта.

Ключевые слова: дифракция; интегральное уравнение; рассеяние радиоволн; радиосвязь; дифракиионная структура; метод моментов; радиоизлучение.

\section{THE DECISION OF FORM FOR DIFFRACTIVE STRUCTURES IN THE PROBLEM OF SCATTERING OF RADIO WAVES}

\section{Preobrazhensky A.P., Choporov O.N.}

This paper considers the problem of scattering of electromagnetic waves in different diffraction structures. The solution of the scattering problem is based on the method of integral equations. On diagrams of backscattering at various frequencies of the incident wave, the decision about the form of the object is carried out.

Keywords: diffraction; integral equation; scattering of radio waves; telecommunication; diffraction structure; method of moments; radioirradiation. 
Для того, чтобы проектировать и оценивать эффективность современных радиолокационных систем требуется знать априорные характеристики рассеяния исследуемых объектов [1-4]. Вследствие развития техники и способов обработки радиолокационных сигналов возникают возможности для увеличения числа и точности измеряемых параметров поля, которое рассеивается от объектов при рассмотрении процессов радиолокации. Следует отметить, что актуальной является задача, связанная с разработкой новых подходов и алгоритмов, направленных на обнаружение и селекцию радиолокационных сигналов [5-8].

Информацию, связанную с состояниемпринимаемых электромагнитных волн можно использовать для того, чтобы обнаруживать и проводить селекцию радиосигналов по соответствующим признакам [7].

Идентификация объектов в радиолокационной сфере может осуществляться по спектрам отраженных сигналов и значениям эффективной поверхности рассеяния (ЭПР). По удаленным (точечным) рассеивателям ЭПР рассматривается как мера их видимости для радаров. Так как точных решений задач определения ЭПР для произвольных тел в общем случае не найдено, были разработаны несколько способов ([9-11]) для того, чтобы ее приближенным образом решать: аналитические, численные и комбинированные.

Когда размеры тел являются сопоставимыми с длиной падающей плоской монохромной волны 1 - то мы говорим о зоне резонансного рассеивания здесь эффективными являются метод конечных элементов (МКЭ) в частотной области и метод моментов (MoM).

В данной работе мы рассматриваем задачу об определении формы дифракционной структуры на основе информации о угловых зависимостях ЭПР. Примеры рассматриваемых структур приведены на рис. 1. 
Рассмотрим решение задачи в рамках метода интегральных уравнений. Запишем интегральное уравнение Фредгольма второго рода для электрического тока [12] при учете граничных условий для поверхностей идеально проводящих объектов (рис. 1)

$$
\mathrm{J}_{s}(\mathrm{r})=2 \mathrm{n} \times \mathrm{H}^{i}(\mathrm{r})+\frac{1}{2 \pi} \mathrm{n} \times \int_{s} \mathrm{~J}_{s}(\mathrm{r}) \times \operatorname{grad}^{\prime} G d s^{\prime},
$$

где $G=\exp (-j k r) / r$ - является трехмерной функцией Грина, относящейся к свободному пространству, она будет являться решением уравнения Гельмгольца когда $\delta$-образный источник; $s$ - обозначает поверхность объектов; $n-$ показывает внешнюю нормаль к поверхности объекта для точки наблюдения;

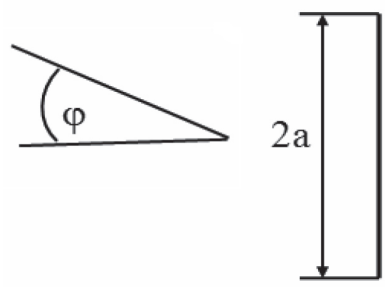

a)

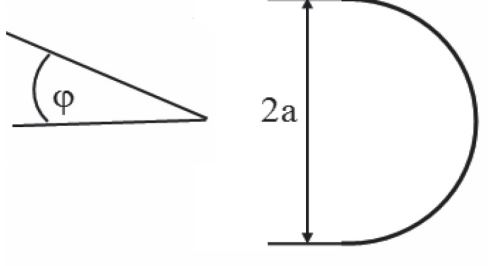

в)

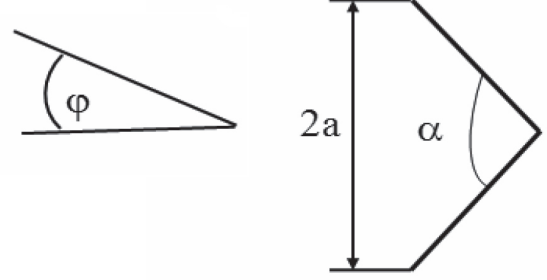

б)

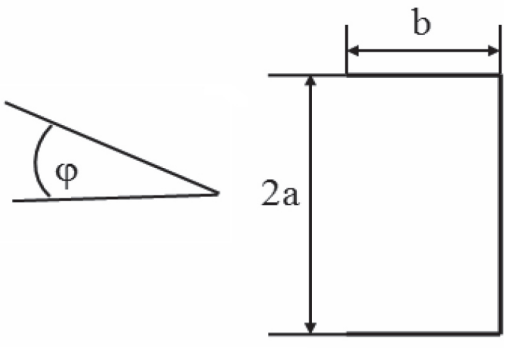

г)

Рис. 1

$J_{S}=[n \times H]-$ является поверхностной плотностью эквивалентного электрического тока; $H^{i}(r)=\mathbf{x} H_{x}^{i}+\mathbf{y} H_{y}^{i}+\mathbf{z} H_{z}^{i}-$ является вектором первичной плоской электромагнитной волны, которая вертикально поляризована. 
Для того, чтобы определить неизвестные поверхностные электрические токи $J_{S}(r)$ на поверхности анализируемых структур проводится решение интегрального уравнения (1) на основе метода моментов [12] таким образом. Определяем систему базисных функций, относительно которых происходит разложение электрических токов на поверхности структур. Для нашего случая в качестве базисных функций мы выбираем кусочно-постоянные функции. Потом требуется ввести систему пробных функций. Пробными функциями в нашем случае были взяты $\delta$-функции Дирака. Применение такой системы с точки зрения физики показывает, что выполнение граничных условий по магнитному полю происходит не для всех поверхностей объектов $S$, а только для дискретного числа точек. Анализ показывает [12], что когда выбирается достаточное количество точек, по которым происходит удовлетворение граничных условий, мы можем получить высокую точность решений, когда осуществляется моделирование рассеяния электромагнитных волн на различных объектах.

Как результат применения метода моментов интегральное уравнение (1) превращается в систему уравнений:

$$
\left[\begin{array}{lll}
U_{x x} & U_{x y} & U_{x z} \\
U_{y x} & U_{y y} & U_{y z} \\
U_{z x} & U_{z y} & U_{z z}
\end{array}\right]\left[\begin{array}{l}
J_{x} \\
J_{y} \\
J_{z}
\end{array}\right]=\left[\begin{array}{l}
R_{x} \\
R_{y} \\
R_{z}
\end{array}\right],
$$

здесь $J_{x}, J_{y}, J_{z}$ - являются компонентами плотности поверхностных электрических токов.

В указанной выше системе для вычисления блоков в матрице $U$ используются следующие выражения:

$$
\begin{gathered}
\left(U_{x x}\right)_{m n}=\frac{1}{2 \pi} \int_{S}\left(\left(n_{y}\right)_{m}\left(\operatorname{grad}_{y}^{\prime}\right)_{m n}+\left(n_{z}\right)_{m}\left(\operatorname{grad}_{z}^{\prime}\right)_{m n}\right) d s_{n}^{\prime}-\delta_{m n} ; \\
\left(U_{x y}\right)_{m n}=-\frac{1}{2 \pi} \int_{S}\left(n_{y}\right)_{m}\left(\operatorname{grad}_{x}^{\prime}\right)_{m n} d s_{n}^{\prime} ;\left(U_{x z}\right)_{m n}=-\frac{1}{2 \pi} \int_{S}\left(n_{z}\right)_{m}\left(\operatorname{grad}_{x}^{\prime}\right)_{m n} d s_{n}^{\prime} ;
\end{gathered}
$$




$$
\begin{gathered}
\left(U_{y x}\right)_{m n}=-\frac{1}{2 \pi} \int_{S}\left(n_{x}\right)_{m}\left(\operatorname{grad}_{y}^{\prime}\right)_{m n} d s_{n}^{\prime} ;\left(U_{y z}\right)_{m n}=-\frac{1}{2 \pi} \int_{S}\left(n_{z}\right)_{m}\left(\operatorname{grad}_{y}^{\prime}\right)_{m n} d s_{n}^{\prime} ; \\
\left(U_{y y}\right)_{m n}=\frac{1}{2 \pi} \int_{S}\left(\left(n_{x}\right)_{m}\left(\operatorname{grad}_{x}^{\prime}\right)_{m n}+\left(n_{z}\right)_{m}\left(\operatorname{grad}_{z}^{\prime}\right)_{m n}\right) d s_{n}^{\prime}-\delta_{m n} ;(3) \\
\left(U_{z x}\right)_{m n}=-\frac{1}{2 \pi} \int_{S}\left(n_{x}\right)_{m}\left(\operatorname{grad}_{z}^{\prime}\right)_{m n} d s_{n}^{\prime} ;\left(U_{z y}\right)_{m n}=-\frac{1}{2 \pi} \int_{S}\left(n_{y}\right)_{m}\left(\operatorname{grad}_{z}^{\prime}\right)_{m n} d s_{n}^{\prime} ; \\
\left(U_{z z}\right)_{m n}=\frac{1}{2 \pi} \int_{S}\left(\left(n_{x}\right)_{m}\left(\operatorname{grad}_{x}^{\prime}\right)_{m n}+\left(n_{y}\right)_{m}\left(\operatorname{grad}_{y}^{\prime}\right)_{m n}\right) d s_{n}^{\prime}-\delta_{m n},
\end{gathered}
$$

где $m, n=1, \ldots, N$, где $N$ - количество точек разбиения поверхности объектов.

Здесь $\mathrm{d}_{m n}$ - является символом Кронекера,

$$
\begin{aligned}
& \operatorname{grad} G_{m n}=-\hat{r}_{m n} \frac{1+j k r}{r_{m n}{ }^{2}} \exp \left(-j k r_{m n}\right)= \\
& \mathbf{i}\left(\operatorname{grad}_{x}\right)_{m n}+\mathbf{j}\left(\operatorname{grad}_{y}\right)_{m n}+k\left(\operatorname{grad}_{z}^{\prime}\right)_{m n},
\end{aligned}
$$

где $\hat{\mathrm{r}}_{m n}=\frac{\mathrm{r}_{m n}}{\left|\mathrm{r}_{m n}\right|}$ - рассчитывается в виде единичного вектора, направленного из точки источника в точку наблюдения [12].

Для вектора-столбца свободных членов записываются такие выражения:

$$
\begin{aligned}
& \left(R_{x}\right)_{m}=2\left(\left(n_{y}\right)_{m}\left(H_{z}^{i}\right)_{m}-\left(n_{z}\right)_{m}\left(H_{y}^{i}\right)_{m}\right) ; \\
& \left(R_{y}\right)_{m}=-2\left(\left(n_{x}\right)_{m}\left(H_{z}^{i}\right)_{m}-\left(n_{z}\right)_{m}\left(H_{x}^{i}\right)_{m}\right) ; \\
& \left(R_{z}\right)_{m}=2\left(\left(n_{x}\right)_{m}\left(H_{y}^{i}\right)_{m}-\left(n_{y}\right)_{m}\left(H_{x}^{i}\right)_{m}\right) .
\end{aligned}
$$

После того, как решена приведенная система уравнений (2) происходит вычисление рассеянного электромагнитного поля для дальней зоны, которое связано с определенным электрическим током $J_{S}(r)$ таким образом[12]:

$$
\mathrm{H}^{s c}(\mathrm{r})=\frac{j \omega \sqrt{\mu_{0} \varepsilon_{0}} \exp (-j k r)}{4 \pi r} \int_{s} \mathrm{~J}_{s}\left(\mathrm{r}^{\prime}\right) \times \hat{\mathrm{r}} \exp \left(j k \hat{\mathrm{r}} \cdot \mathrm{r}^{\prime}\right) d s^{\prime},
$$

где $k$ - является волновым числом;

$\mu_{0}=4 \cdot \pi \cdot 10^{-7} \Gamma \mathrm{H} / \mathrm{M}, \varepsilon_{0}=8,85 \cdot 10^{-12} \Phi / \mathrm{M}-$ представляют собой магнитную и электрическую постоянную; 
$r^{\prime}-$ является радиус-вектором точки источника;

$r$ - является радиус-вектором точки наблюдения по дальней зоне.

Проводится расчет ЭПР объекта на основе такой формулы [13]

$$
\sigma=\lim _{r \rightarrow \infty} 4 \pi r^{2} \frac{\left|\mathrm{H}^{s c}(\mathrm{r})\right|^{2}}{\left|\mathrm{H}^{i}(\mathrm{r})\right|^{2}}
$$

Определение формы объекта может быть полезным в различных автоматизированных системах $[14,15]$. Для того, чтобы принять решение о форме объекта, мы будем рассматривать случаи, когда сначала падает электромагнитная волна с частотой $\mathrm{f}_{1}$, а затем с частотой $\mathrm{f}_{2}$, причем их значение отличается на порядок.

Характеристика рассеяния объекта, приведенного на рис 1a, в обоих случаях будет иметь характерный максимум при нулевом угле наблюдения.

Объекты, приведенные на рис 16 и 1г, по характеристикам рассеяния отличаются тем, что во втором случае их значения отличаются на большие величины, что позволяет принять решение, что объектом является полая прямоугольная структура. При относительно небольших изменениях (не более 5-6 дБ) для сектора углов $0^{\circ}-20^{\circ}$ принимается решение, что рассматривается структура, приведенная на рис. 16.

Для того, чтобы принять решение о том, что мы рассматриваем объект, приведенный на рис. 1в, необходимо провести анализ изменений характеристик рассеяния в секторе углов наблюдения $0^{\circ}-30^{\circ}$. Если значения изменяются не более, чем на 2-3 дБ, то мы принимаем решение.

\section{Вывод}

В работе рассмотрены вопросы принятия решения о форме объектов на основе анализа характеристик рассеяния при различных частотах падающей волны. 


\section{Список литературы}

1. Головинов С.О., Хромых А.А. Проблемы управления системами мобильной связи // Вестник Воронежского института высоких технологий. 2012. № 9. С. 13-14.

2. Казаков Е.Н. Разработка и программная реализации алгоритма оценки уровня сигнала в сети wi-fi // Моделирование, оптимизация и информационные технологии. 2016. № 1. С. 13.

3. Пекшев Г.А., Скляр А.Г. Некоторые свойства лучевых методов, используемых для анализа распространения электромагнитных волн // В мире научных открытий. 2015. № 12. С. 17-23.

4. Пекшев Г.А., Скляр А.Г. Проблемы управления рассеянными электромагнитными полями // В мире научных открытий. 2015. № 12. C. 24-30.

5. Максимова А.А., Юрочкин А.Г. Методы исследования характеристик рассеяния электромагнитных волн объектами // Вестник Воронежского института высоких технологий. 2016. № 16. С. 53-56.

6. Канавин С.В., Лукьянов А.С. Перспективы применения систем мобильного широкополосного доступа в сетях подвижной радиосвязи на основе стандартов mobile WIMAX и LTE // Вестник Воронежского института высоких технологий. 2016. № 16. С. 79-82.

7. Кульнева Е.Ю., Гащенко И.А. О характеристиках, влияющих на моделирование радиотехнических устройств // Современные наукоемкие технологии. 2014. № 5-2. С. 50.

8. Глотова Т.В. Применение гибридного метода для расчета характеристик рассеяния объектов над шероховатой поверхностью // Моделирование, оптимизация и информационные технологии. 2016. № 1. С. 11.

9. Баранов А.В. Проблемы функционирования mesh-сетей // Вестник Воронежского института высоких технологий. 2012. № 9. C. $49-50$. 
10.Милошенко О.В. Методы оценки характеристик распространения радиоволн в системах подвижной радиосвязи // Вестник Воронежского института высоких технологий. 2012. № 9. C. $60-62$.

11. Ерасов С.В. Оптимизационные процессы в электродинамических задачах // Вестник Воронежского института высоких технологий. 2013. № 10. C. 20-26.

12.Щербатых С.С. Метод интегральных уравнений как основной способ анализа в САПР антенн // Моделирование, оптимизация и информационные технологии. 2016. № 1. С. 10.

13. Справочник по радиолокации / Под ред. Сколника М. М.: Сов. радио, 1976. Т.1. $455 \mathrm{c.}$

14. Мишин Я.А. О системах автоматизированного проектирования в беспроводных сетях // Вестник Воронежского института высоких технологий. 2013. № 10. С. 153-156.

15. Болучевская О.А., Горбенко О.Н. Свойства методов оценки характеристик рассеяния электромагнитных волн // Моделирование, оптимизация и информационные технологии. 2013. № 3. С. 4.

\section{References}

1. Golovinov S.O., Khromykh A.A. Vestnik Voronezhskogo instituta vysokikh tekhnologiy. 2012. № 9, pp. 13-14.

2. Kazakov E.N. Modelirovanie, optimizatsiya i informatsionnye tekhnologii. 2016. № 1. P. 13.

3. Pekshev G.A., Sklyar A.G. Vmire nauchnykh otkrytiy. 2015. № 12, pp. 17-23.

4. Pekshev G.A., Sklyar A.G. V mire nauchnykh otkrytiy. 2015. № 12, pp. 24-30.

5. Maksimova A.A., Yurochkin A.G. Vestnik Voronezhskogo instituta vysokikh tekhnologiy. 2016. № 16, pp. 53-56.

6. Kanavin S.V., Luk'yanov A.S. Vestnik Voronezhskogo instituta vysokikh tekhnologiy. 2016. № 16, pp. 79-82. 
7. Kul'neva E.Yu., Gashchenko I.A. Sovremennye naukoemkie tekhnologii. 2014. № 5-2. P. 50.

8. Glotova T.V. Modelirovanie, optimizatsiya i informatsionnye tekhnologii. 2016. № 1. P. 11.

9. Baranov A.V. Vestnik Voronezhskogo instituta vysokikh tekhnologiy. 2012. № 9, pp. 49-50.

10.Miloshenko O.V. Vestnik Voronezhskogo instituta vysokikh tekhnologiy. 2012. № 9, pp. 60-62.

11.Erasov S.V. Vestnik Voronezhskogo instituta vysokikh tekhnologiy. 2013. № 10, pp. 20-26.

12. Shcherbatykh S.S. Modelirovanie, optimizatsiya i informatsionnye tekhnologii. 2016. № 1. P. 10.

13.Spravochnik po radiolokatsii [Reference radar] / Skolnik M. (ed.). M.: Sov. ra-dio, 1976. t.1. 455 p.

14.Mishin Ya.A. Vestnik Voronezhskogo instituta vysokikh tekhnologiy. 2013. № 10, pp. 153-156.

15.Boluchevskaya O.A., Gorbenko O.N. Modelirovanie, optimizatsiya i informatsionnye tekhnologii. 2013. № 3. P. 4.

\section{ДАННЫЕ ОБ АВТОРАХ}

\section{Преображенский А.П., Чопоров О.Н.}

Воронежский институт высоких технологий

ул. Ленина, 73А, Воронеж, Воронежская обл., 394043, Российская Федерация

komkovvivt@ya.ru

\section{DATA ABOUT THE AUTHORS \\ Preobrazhensky A.P., Choporov O.N.}

Voronezh Institute of High Technologies

73A, Lenin Str., Voronezh region, 394043, Russian Federation

komkovvivt@ya.ru 